\title{
Probing the atomic structure of metallic nanoclusters with the tip of a scanning tunneling microscope
}

\author{
Koen Schouteden, ${ }^{\text {*a }}$ Koen Lauwaet, $\dagger^{\mathrm{a}}$ Ewald Janssens, ${ }^{a}$ Giovanni Barcaro, ${ }^{\text {*b }}$ \\ Alessandro Fortunelli, ${ }^{b}$ Chris Van Haesendonck $^{a}$ and Peter Lievens ${ }^{a}$ \\ Preformed Co clusters with an average diameter of $2.5 \mathrm{~nm}$ are produced in the gas phase and are deposited \\ under controlled ultra-high vacuum conditions onto a thin insulating $\mathrm{NaCl}$ film on $\mathrm{Au}(111)$. Relying on a \\ combined experimental and theoretical investigation, we demonstrate visualization of the three- \\ dimensional atomic structure of the Co clusters by high-resolution scanning tunneling microscopy (STM) \\ using a $\mathrm{Cl}$ functionalized STM tip that can be obtained on the $\mathrm{NaCl}$ surface. More generally, use of a \\ functionalized STM tip may allow for systematic atomic structure determination with STM of \\ nanoparticles that are deposited on metal surfaces.
}

Received 12th July 2013

Accepted 26th November 2013

DOI: $10.1039 / c 3 n r 03585 a$

www.rsc.org/nanoscale

\section{Introduction}

The undiminished research in the field of nanotechnology has led to a diversity of routes that allow controlled preparation of nanoparticles out of virtually any material and exhibiting virtually any possible size and shape. Because of their broad range of unique and tunable properties that are related to the emergence of quantum size effects, nanoparticles offer only partially exploited potential to build new electronic, optical and chemically sensitive devices. For this purpose, nanoparticles are grown, ${ }^{1-3}$ deposited $^{4,5}$ and manipulated ${ }^{6,7}$ on the desired surfaces. To fully exploit their potential, detailed knowledge of the structure of the individual nanoparticles down to the atomic level is obviously required, allowing, e.g., the determination of active catalytic sites on nanoparticles. ${ }^{1,48}$ Visualization of the structure of nanoparticles with atomic resolution remains, however, experimentally challenging.

The continuous development of transmission electron microscopy techniques currently provides a lateral resolution as high as $50 \mathrm{pm} .{ }^{5}$ However, it is still not straightforward to obtain height information to determine the three-dimensional (3D) geometry of nanoparticles. ${ }^{9,10}$ In particular, particles may rotate or suffer from structural changes due to irradiation with the electron beam. ${ }^{5}$ Scanning probe microscopy is in principle able to provide height information of the nanoparticle surface with atomic precision, in particular when working in a ultra-high vacuum (UHV) and

\footnotetext{
${ }^{a}$ Laboratory of Solid-State Physics and Magnetism, KU Leuven, Celestijnenlaan 200 D, BE-3001 Leuven, Belgium. E-mail: Koen.Schouteden@fys.kuleuven.be; Fax: +32 16 327983; Tel: +3216327245

${ }^{b}$ CNR-IPCF, Istituto per i Processi Chimico-Fisici del CNR, via Giovanni Moruzzi 1, I56124 Pisa, Italy.E-mail: barcaro@pi.ipcf.cnr.it

$\dagger$ Present address: IMDEA Nanociencia, C/Faraday, 9, Campus Universitario de Cantoblanco, 28049 Madrid, Spain.
}

at low temperatures. The possibility to perform scanning tunneling microscopy (STM) imaging simultaneously with scanning tunneling spectroscopy (STS) offers unique opportunities to study the morphology and electronic properties with very high spatial and energy resolution. However, the number of reported STM results that achieved to some extent imaging of the 3D structure of nanoparticles with atomic resolution remains very limited. ${ }^{11-14}$ This can be mainly related to tip convolution effects that exist due to the finite size of the tip apex and that hamper detailed imaging, in particular of non-planar objects. Therefore, an exceptionally sharp tip is required for atomic resolution imaging of the (typically non-planar) nanoparticle surface, implying that systematic atomic resolution investigation cannot be performed.

Here, we demonstrate that atomic resolution imaging of quasi-spherical nanometer size metallic particles (Co) on metallic surfaces $[\mathrm{Au}(111)]$ can be achieved upon covering the substrate with a thin insulating film $(\mathrm{NaCl})$. Via a combined analysis of STM measurements and Coulomb-blockade related energy gaps observed in STS spectra, we find that the Co clusters penetrate (at least) partially into the $\mathrm{NaCl}$ layer due to impact upon deposition. Functionalization of the STM tip on the $\mathrm{NaCl}$ surface $^{15-17}$ yields unprecedented atomic-scale resolution images based on which a 3D atomic model of the deposited Co cluster can be constructed. This is complemented by a novel density-functional-theory-based model that quantitatively demonstrates the influence of the STM tip apex on the visualization of the 3D structure of the clusters.

\section{Methods section}

\subsection{Sample preparation and STM/STS measurements}

A description of the STM setup (Omicron Nanotechnology) and details on the preparation of the $\mathrm{Au}(111)$ surface and the 
tungsten STM tips are given in ref. 29. NaCl (99.999\% purity, Alfa Aesar) is sublimated at $800 \mathrm{~K}$ with the substrate kept at room temperature, after which the sample is annealed to obtain trilayer $\mathrm{NaCl}^{20}$ All STM measurements are performed at $4.5 \mathrm{~K}$. Image processing is performed by Nanotec WSxM. ${ }^{30}$ Co clusters are produced by a laser vaporization source, ${ }^{26,31,32}$ which is connected to a UHV cluster deposition chamber with a base pressure below $5 \times 10^{-10} \mathrm{mbar}$. The sample is transferred from and to the STM setup by means of a UHV transport vessel (pressure in the $10^{-10}$ mbar range).

\subsection{Theoretical background}

Density functional theory (DFT) calculations are performed on various structural models of Co clusters deposited on a bare $\mathrm{Au}(111)$ surface and on a $\mathrm{Au}(111)$ surface covered by a trilayer of $\mathrm{NaCl}(100)$. A plane-wave basis set and the GGA-PBE xc-correlation functional ${ }^{33}$ are used as implemented in the Quantum Espresso package; ${ }^{34} 40$ Ry and 240 Ry are chosen as the energy cutoff values for the selection of the plane waves to describe the wave function and the electronic density, respectively. The $\mathrm{Au}(111)$ surface is modeled as a slab made by two layers frozen in crystal positions according to an fcc lattice characterized by the experimental lattice constant of gold (4.08 $\mathrm{A})$. The $\mathrm{NaCl}(100)$ surface is modeled as a slab made by three layers frozen in crystal positions according to an fcc lattice (of both the $\mathrm{Na}^{+}$and $\mathrm{Cl}^{-}$ions) characterized by the experimental lattice constant of bulk NaCl (3.96 ̊). A tentative atomistic structure of the Co clusters is derived from the analysis of experimental images, and is then used as a starting point of local energy minimizations. The atoms of the Co cluster are allowed to relax while keeping the $\mathrm{Au}(111)$ and $\mathrm{NaCl}(100)$ frozen until the forces on the metal cluster are smaller than $0.01 \mathrm{eV} \AA^{-1}$.

The simulation of STM images of Co clusters on $\mathrm{NaCl}-\mathrm{Au}(111)$ is conducted in two steps. First, the limit of an infinitely sharp (point) tip is assumed and DFT calculations are performed using a Tersoff-Hamann approach. ${ }^{35}$ Second, the effect of the atomistic structure of the tip on the STM images is investigated using an effective model whose parameters are extracted from the previous DFT simulations. Assuming that each pair of atoms in the sample-tip system contributes to the tunneling current with an additive term which only depends on the types of atoms and their spatial distance, the current is given by:

$$
I=\sum_{i j} A_{i} \exp \left(-B_{j} r_{i j}\right)
$$

where $r_{i j}$ is the distance between the atom $i$ of the STM tip and the atom $j$ of the sample and $A_{j}$ and $B_{j}$ are two parameters that depend on the nature of the atom $j .{ }^{36}$ Constant-current STM images are then easily constructed by plotting contour surfaces of $I$. Note that in this model specific band structure effects ${ }^{37}$ are not taken into account since they should not be crucial in conductive systems as the ones considered here. Using eqn (1), both the shape and the size of the tip apex can be easily adjusted, thereby going beyond the standard Tersoff-Hamann model of an infinitely sharp tip.

\section{Results and discussion}

\subsection{Morphology and electronic decoupling of self-pinned Co clusters}

Fig. 1(a) and (b) present STM topography images of preformed Co clusters that are deposited using a molecular beam on $\mathrm{Au}(111)$ and $\mathrm{NaCl}-\mathrm{Au}(111)$. The herringbone reconstruction can be resolved on both surfaces. ${ }^{17}$ It can be seen that the clusters are randomly distributed across the $\mathrm{Au}(111)$ and $\mathrm{NaCl}-\mathrm{Au}(111)$ surfaces. This implies that the clusters are pinned upon impact due to the inherent kinetic energy of the clusters in the molecular beam. ${ }^{18} \mathrm{On} \mathrm{NaCl}-\mathrm{Au}(111)$, self-pinning is accompanied by local damaging of the $\mathrm{NaCl}$ film, which is evidenced by the presence of atomic size defects next to the clusters (data not shown). As will be discussed below, this indirectly has major implications for the visualization of the Co cluster on NaCl-Au(111) with STM.

Based on height profiles taken from a series of STM topography images recorded at $-1 \mathrm{~V}$ and $0.2 \mathrm{nA}$, we determined the height distribution of the Co clusters. The cluster height is measured with respect to the neighboring surface, i.e., either $\mathrm{Au}(111)$ or $\mathrm{NaCl}-\mathrm{Au}(111)$ (about 100 clusters were measured for each surface). The resulting histograms are presented in Fig. 1(c), together with the diameter distribution of the Co clusters in the molecular beam prior to deposition. ${ }^{19}$ The average cluster heights are $1.9 \pm 0.1 \mathrm{~nm}$ on $\mathrm{Au}(111)$ and $1.7 \pm$ $0.1 \mathrm{~nm}$ on $\mathrm{NaCl}-\mathrm{Au}(111)$, while the average diameter prior to
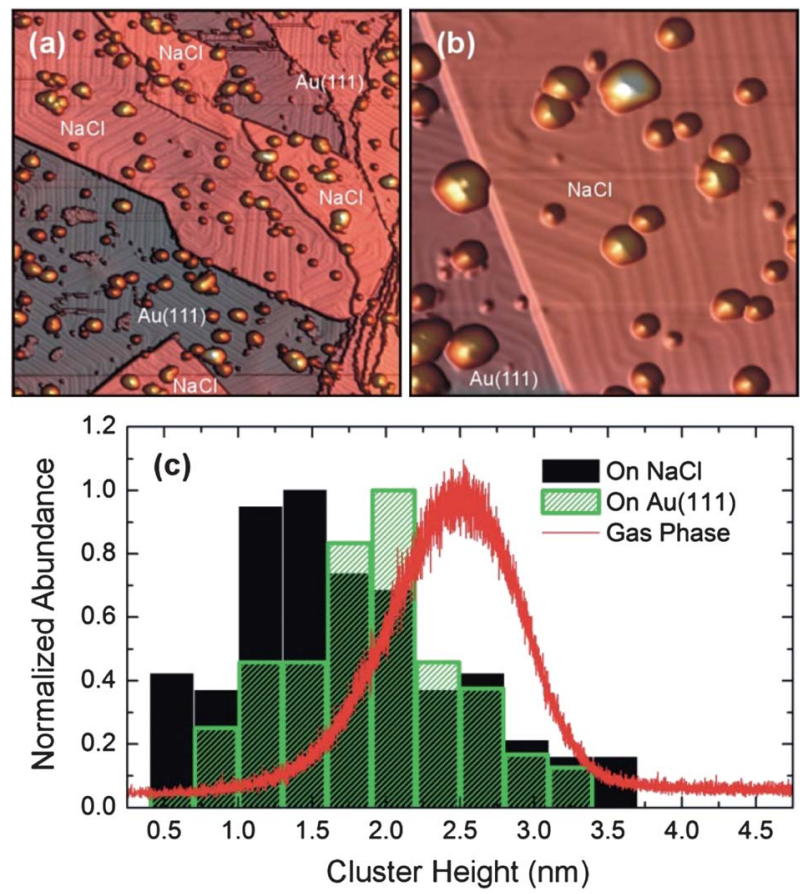

Fig. 1 (a) $260 \times 260 \mathrm{~nm}^{2}$ and (b) $90 \times 90 \mathrm{~nm}^{2}$ 3D rendered STM topography image of $\mathrm{Co}$ clusters on $\mathrm{Au}(111)$ and on $\mathrm{NaCl}-\mathrm{Au}(111)$. (c) Height histogram of the deposited Co clusters on $\mathrm{Au}(111)$ and on $\mathrm{NaCl}-\mathrm{Au}(111)$ determined from STM topography images, together with the diameter abundance spectrum of the clusters in the gas phase prior to deposition. 
deposition is $2.5 \mathrm{~nm}$. We note that the intensity of the largest clusters in the gas phase diameter distribution may be underestimated because of a reduced detection efficiency for heavy particles.

Fig. 2(a) and (b) present typical $I(V)$ and $(\mathrm{d} I / \mathrm{d} V)(V)$ spectra, respectively, recorded on top of two different Co clusters on $\mathrm{NaCl}-\mathrm{Au}(111)$ [see the inset of Fig. 2(a)]. In addition, a spectrum recorded on the $\mathrm{NaCl}-\mathrm{Au}(111)$ surface [labeled (1) in Fig. 2(a) and (b)] is added as a reference. The $(\mathrm{d} I / \mathrm{d} V)(V)$ reference spectrum reveals the step-like onset of the $\mathrm{NaCl}-\mathrm{Au}(111)$ interface state. ${ }^{17,20}$ Surprisingly, the majority of the clusters on $\mathrm{NaCl}-$ $\mathrm{Au}(111)$ (about 90\%) is found to exhibit metallic behavior, such as the cluster labeled (2) in Fig. 2(a) and (b). This implies that the majority of the clusters on $\mathrm{NaCl}-\mathrm{Au}(111)$ penetrates into the $\mathrm{NaCl}$ film sufficiently deep to be in direct electrical contact with the underlying $\mathrm{Au}(111)$ surface.

A minority of the clusters (about $10 \%$ ) exhibits a pronounced gap around zero bias voltage similar to the spectrum labeled (3) in Fig. 2(a) and (b). Fig. 2(c) presents the relationship between the measured cluster height $h$ (with respect to the surrounding $\mathrm{NaCl}$ ) and the gap size $V_{\text {gap }}$ for those clusters that show a gap. ${ }^{21}$ The observed gap can be attributed to Coulomb blocking of the tunneling current that occurs for metallic particles in a doublebarrier tunneling junction. ${ }^{22}$ Assuming - for simplicity - a hemispherical cluster shape, an approximate value of the Coulomb gap can be calculated as a function of the cluster height for clusters on mono-, bi-, and trilayer $\mathrm{NaCl}$ [see Fig. 2(c)]. ${ }^{23}$ The theoretical values of $322 \mathrm{pm}, 601 \mathrm{pm}$ and 876 pm (ref. 24) were used for the thicknesses of mono-, bi- and trilayer $\mathrm{NaCl}$, respectively. It can be seen that the experimental

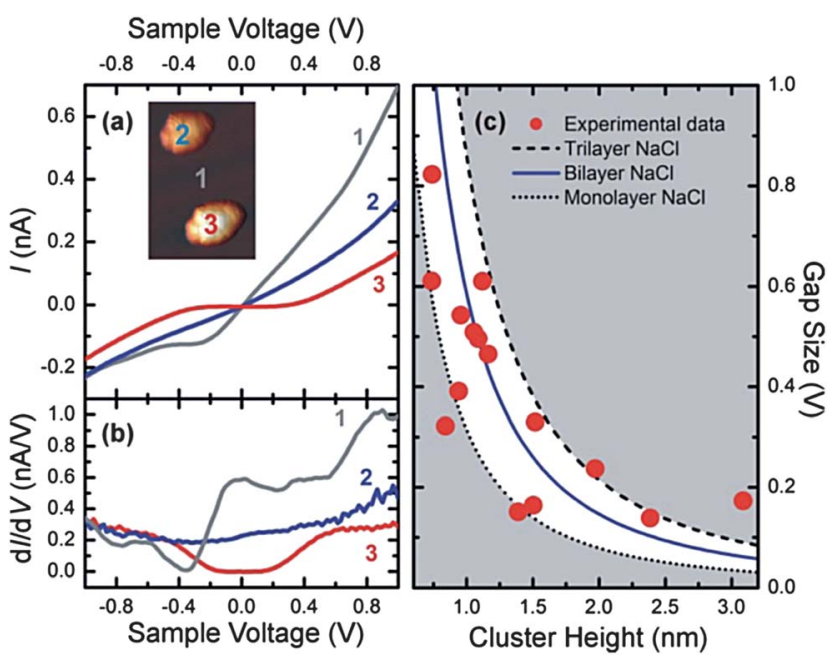

Fig. 2 (a) $I(V)$ and (b) corresponding $(d / / d V)(V)$ spectra taken at the locations indicated in the inset of (a). The majority of the clusters exhibits metallic behavior [cluster (2)], while a minority reveals a pronounced gap in the spectra [cluster (3)]. Inset in (a): STM topography image of two $\mathrm{Co}$ clusters on $\mathrm{NaCl}-\mathrm{Au}(111)$. (c) Size of the Coulomb gap observed in $(\mathrm{d} / / \mathrm{d} V)(V)$ spectra taken on Co clusters versus the height of the Co clusters. The dotted, solid and dashed lines give the dependence of the gap expected for hemispherical clusters on mono-, bi- and trilayer $\mathrm{NaCl}$, respectively, following the parallel plate capacitance model. data points are spread around the bilayer curve and are mostly in between the calculated curves for mono- and trilayer $\mathrm{NaCl}$.

The penetration of a fraction of the Co clusters implies that the height histogram of the Co clusters on NaCl-Au(111) in Fig. 1(c) should have a bimodal character. Indeed, upon more careful inspection of Fig. 1(c), it can be seen that the STM based height histogram measured on $\mathrm{NaCl}-\mathrm{Au}(111)$ has a similar tail as the height histogram measured on bare $\mathrm{Au}(111)$. This confirms that a fraction of the deposited clusters does not fully penetrate into the $\mathrm{NaCl}$ film, which is in agreement with the Coulomb gaps observed in STS spectra. The observed average height of the clusters which are embedded in the $\mathrm{NaCl}$ film is underestimated and should to a first approximation be augmented by the height of trilayer $\mathrm{NaCl}$ on $\mathrm{Au}(111)$ that is measured in STM topography images (0.4 nm (ref. 20)), yielding an average height for the first maximum of about $1.9 \mathrm{~nm}$, i.e. coincident with that for Co clusters on the bare $\mathrm{Au}(111)$. On the other hand, taking into account (limited) deformation of the $\mathrm{NaCl}$ layer for all deposited clusters, the above indicated similar tail in the height histogram implies that the Co clusters experience less flattening on $\mathrm{NaCl}-\mathrm{Au}(111)$ and that they retain to a larger extent their intrinsic spherical shape due to the reduced interaction with the $\mathrm{NaCl}$ film with respect to $\mathrm{Au}(111){ }^{25,26}$

\subsection{Atomic resolution imaging}

Fig. 3(a-c) present high-resolution STM topography images of three different Co clusters on $\mathrm{NaCl}-\mathrm{Au}(111)$. The clusters in (a) and (c) are in contact with the $\mathrm{Au}(111)$ surface and exhibit metallic behavior, while the cluster in (b) only partially penetrated into the $\mathrm{NaCl}$ layer. Remarkably, apart from the typical 1 $\times 1$ atomic structure of the $\mathrm{NaCl}$ surface, clear atomic features can also be discerned on the Co cluster surface. This observation is in contrast to Co clusters on bare $\mathrm{Au}(111)$, for which we were not able to resolve the atomic structure. ${ }^{26}$ It is generally believed that tip convolution effects, inherent to STM imaging, make it extremely challenging or quasi-impossible to obtain atomic resolution on spherical nanoparticles deposited on a metallic surface, ${ }^{\mathbf{1 1}, \mathbf{1 4}}$ implying that reliable structural STM investigation of non-planar nanoparticles cannot be performed down to the atomic scale.

The enhanced resolution of Co clusters on $\mathrm{NaCl}-\mathrm{Au}(111)$ observed here can be related to a functionalization of the STM tip, i.e. picking up of $\mathrm{a} \mathrm{Cl}^{-}$ion with the STM tip. Here, picking up of $\mathrm{a} \mathrm{Cl}^{-}$ion with the STM tip is achieved by scanning the locally damaged $\mathrm{NaCl}$ film and is evidenced by the accompanying drastic enhancement of the imaging resolution. Gross et al. have recently revealed both experimentally and theoretically that an STM tip terminated with a CO molecule yields STM images with greatly enhanced resolution. Such functionalized tips probe the modulus squared of the lateral gradient of the wave functions instead of the modulus squared of the wave functions that is visualized with a bare metallic STM tip. ${ }^{16}$ Similar enhancement of the resolution can be achieved using a Cl terminated tip and even a pentacene molecule terminated tip. ${ }^{15}$ Recently, we achieved enhanced resolution on $\mathrm{NaCl}-$ $\mathrm{Au}(111)$ surfaces by using a $\mathrm{Cl}$ functionalized tip that was 

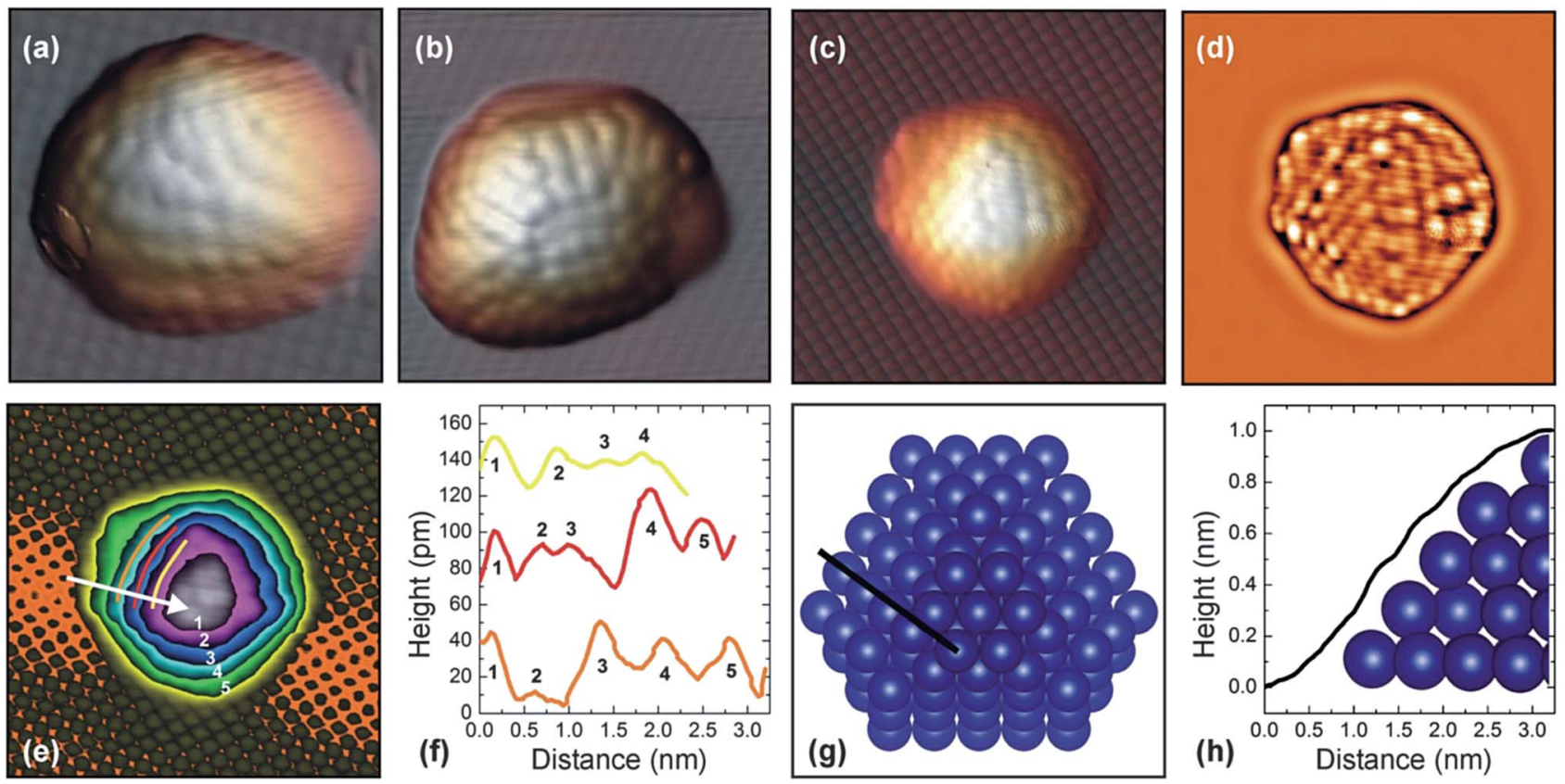

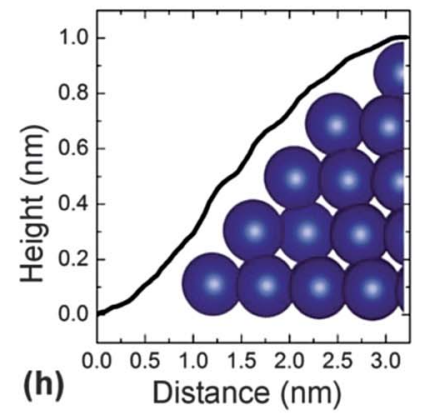

Fig. $3(\mathrm{a}-\mathrm{c})$ Atomic resolution STM topography image of three different Co clusters on $\mathrm{NaCl}-\mathrm{Au}(111)$. Figure sizes are $7 \times 7 \mathrm{~nm}{ }^{2}$, (b) $8 \times 8 \mathrm{~nm}{ }^{2}$ and (c) $9 \times 9 \mathrm{~nm}^{2}$, respectively. Tunneling settings are (a) $-1.3 \mathrm{~V}$ and $0.65 \mathrm{nA}$, (b) $-1.5 \mathrm{~V}$ and $0.3 \mathrm{nA}$, (c) $-1.5 \mathrm{~V}$ and $2.0 \mathrm{nA}$. (d) Same as in (c) after applying Fourier filtering to better resolve the atoms of the cluster surface. (e) Same image as in (c) with an adjusted color scale to better visualize the subsequently stacked atomic layers of the cluster. (f) Height profiles taken along the colored lines in (e). Profiles are given an offset for clarity. Note that the lateral interatomic distances are overestimated due to tip convolution effects that laterally broaden the (hemi)spherical cluster surface. (g) Top view of a ball model of the Co cluster, constructed based on the atomic resolution image in (c). (h) Height profile taken along the white arrow in (e), together with the corresponding cross-section of the atomistic cluster model [indicated by the black line in (g)]. The model is arbitrarily stretched along the horizontal axis to reflect the experimental lateral broadening.

created by scanning the $\mathrm{NaCl}$ surface in close proximity until a $\mathrm{Cl}^{-}$ion was picked up. ${ }^{17} \mathrm{Here}$, the probability of such an event is increased significantly due to the fact that the $\mathrm{NaCl}$ film is locally damaged by the impact of the deposited Co clusters. This way, atomic resolution imaging of Co clusters on $\mathrm{NaCl}-\mathrm{Au}(111)$ can be routinely achieved, as illustrated in Fig. $3(\mathrm{a}-\mathrm{c})$.

In the following we focus on the Co cluster in Fig. 3(c) and construct a 3D model of this cluster based on the observed atomic structure and the height information in the STM topography image. Clusters that are not in contact with the $\mathrm{Au}(111)$ surface can have any possible orientation with respect to the $\mathrm{Au}(111)$ surface. On the other hand, clusters that are in contact with the metallic $\mathrm{Au}(111)$ surface adapt to a certain extent their orientation and crystal structure to the $\mathrm{Au}(111)$ surface, i.e. the atomic layers are typically oriented parallel to the $\mathrm{Au}(111)$ surface..$^{25,26}$

From the measured height of the cluster in Fig. 3(c) with respect to the surrounding $\mathrm{NaCl}$, we infer that the cluster consists of 7 atomic layers, i.e. 5 layers appear above the surrounding $\mathrm{NaCl}$ film and 2 layers are below the $\mathrm{NaCl}$ film surface. This layered structure allows us to assign the observed atomic structure in Fig. 3(c) to different atomic layers. For this purpose, the experimental image was Fourier filtered to better resolve the atoms at the cluster surface [see Fig. 3(d)] and, in addition, the color code was adjusted to link the observed atoms to different atomic layers [see Fig. 3(e)]. Each color of the cluster (green, light blue, dark blue, purple, and grey) in Fig. 3(e) corresponds to an atomic layer. Upon careful inspection of Fig. 3(c-e) it can be seen that the atoms of the top facet of the cluster [grey color in Fig. 3(e)] appear to have a hexagonal arrangement. While small Co particles typically exhibit an fcc crystal structure, ${ }^{27}$ it is known that an hep growth on fcc facets can be kinetically stabilized ${ }^{28}$ similar to what occurs, e.g., during growth of icosahedra from smaller decahedra. Fig. 3(f) presents three height profiles taken at different atomic layers of the cluster [indicated by colored lines in Fig. 3(e)], revealing the positions of the individual atoms in these layers. Note that the lateral interatomic distances in the height profiles are overestimated due to unavoidable convolution effects related to the finite size of the STM tip. Tip convolution laterally broadens the (hemi)spherical cluster surface in STM topography images and consequently increases the apparent interatomic distances at the probed cluster surface as can be seen in Fig. 3(e).$^{40}$ The distances between the probed surface atoms of the atomically flat $\mathrm{NaCl}$ layer obviously do not suffer from tip convolution. The height of the cluster remains unaffected as well. We find that the observed atomic structure of the Co cluster can be matched qualitatively with that of a cluster having an fcc structure and with an hep arrangement of the top facet atoms.

A 3D atomistic model of the Co cluster is presented in Fig. $3(\mathrm{~g})$. Finally, Fig. $3(\mathrm{~h})$ presents a cross-section of (the 5 topmost atomic layers of) the 3D model together with the corresponding experimental height profile. Assuming a distance of $0.20 \pm 0.01 \mathrm{~nm}$ between the stacked Co layers, ${ }^{26,41}$ our cluster 
model correctly reproduces the measured cluster height in Fig. 3(h). On the other hand, the model is arbitrarily stretched along the horizontal axis to reflect the lateral broadening of the imaged cluster surface due to tip convolution effects. Taking into account the bulk lateral distance between Co atoms of $0.25 \mathrm{~nm}$, the tip convolution effects result in a lateral broadening by a factor of about 2 . Though more complicated, the procedure employed here may be applied as well for clusters that only partially penetrate into the $\mathrm{NaCl}$ layer by taking into account the different possible orientations of the cluster atomic layers with respect to the $\mathrm{Au}(111)$ surface.

\subsection{Simulation of STM images}

We performed theoretical simulations of STM images to investigate in more detail the role of the size and shape of the STM tip apex in the resolution obtained in STM images of Co clusters on bare $\mathrm{Au}(111)$ and on $\mathrm{NaCl}-\mathrm{Au}(111)$. The geometry of the Co cluster in Fig. 3(g) after DFT relaxation on the $\mathrm{Au}(111)$ surface is presented in Fig. 4(a) and (b). While tip convolution effects have been the subject of numerous investigations, the effect of the size and shape of the STM tip on the atomic corrugation of nonplanar surfaces has to the best of our knowledge not been studied before down to the atomic level. The high amount (several hundreds) of atoms involved (the Co cluster, the NaCl trilayer, the $\mathrm{Au}(111)$ surface and the STM tip) make a full ab initio calculation very demanding and beyond the scope of the present manuscript. Therefore, we developed a versatile effective model based on DFT calculations that allows us to conveniently simulate the effect of the atomic structure of the tip.

Regarding the atomistic model employed to simulate the STM images, we cut a rectangular portion of a (111) surface of gold, extending about $35 \times 98 \AA^{2}$ in the $x y$ plane; one half of this surface was covered by three layers of $\mathrm{NaCl}$ according to a stacking of (100) planes. On both the uncovered and the $\mathrm{NaCl}-$ covered Au surfaces two identical Co clusters were adsorbed: each cluster was composed of 7 (111) layers in fcc stacking (except for the top-most one, positioned in hcp stacking) and corresponded to a surface containing a total of 118 atoms (in our simulations, only the surface atoms contributed to the STM signal).

The considered tips consist of 1 to 200 atoms and an increase of the cluster dimension (lateral broadening) and a decrease of the resolution with increasing tip size were observed as expected. These effects converge at a certain tip size. This, however, depends crucially on the tip shape. In particular, tip effects reach convergence or stabilize for tips of above 50 and 100 atoms for a tetrahedral and a pyramidal tip shape, respectively, which can be related to the symmetry of the tip with respect to the cluster.

Fig. 4(c) and (d) present simulated STM images of the Co clusters in Fig. 4(a) and (b), obtained using the empirical model with (c) a blunt pyramidal STM tip consisting of 12 atoms and (d) an atomically sharp STM tip consisting of 13 atoms. Broadening due to tip convolution is highlighted by dashed lines in Fig. 4(a-c). We note that in the experiment the STM tip can be more blunt and it may also have an asymmetric shape, leading to even more pronounced broadening and deformation effects.

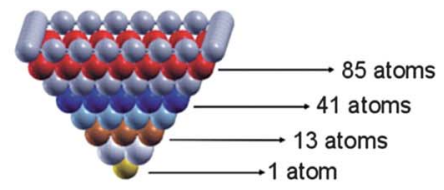

(a)

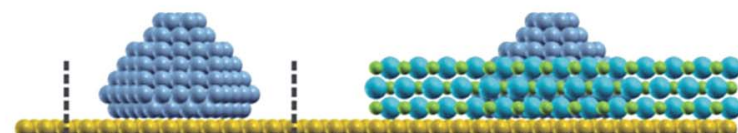

(b)

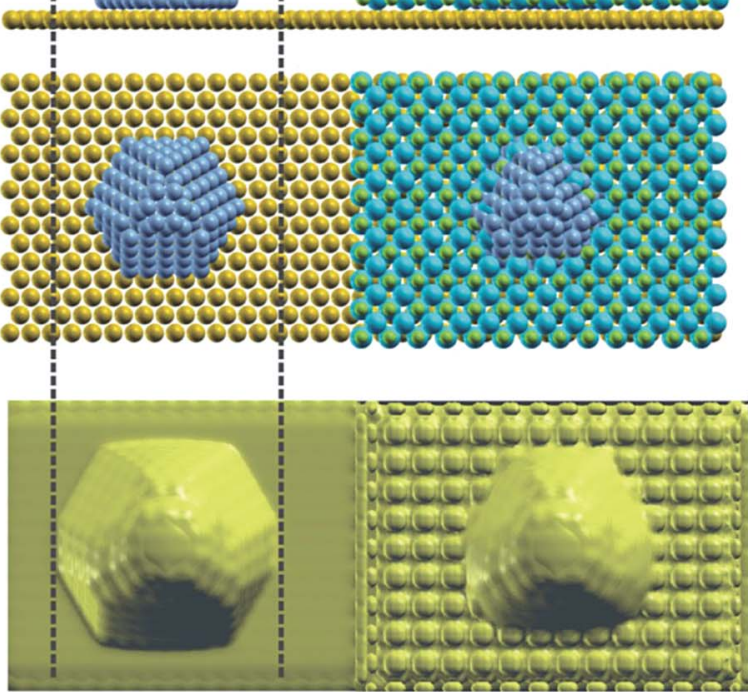

(d)

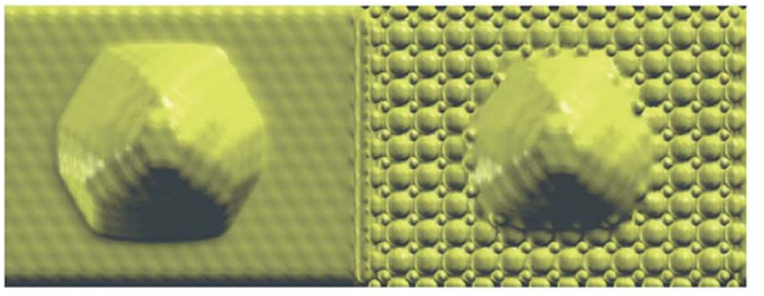

Fig. 4 Side view (a) and top view (b) of the atomistic model of a Co cluster on bare $\mathrm{Au}(111)$ (left) and on $\mathrm{NaCl}-\mathrm{Au}$ (111) (right) (yellow: $\mathrm{Au}$ atoms; cyan: $\mathrm{Na}$ atoms; green: $\mathrm{Cl}$ atoms; dark blue: Co atoms). An atomistic model of a pyramidally shaped STM tip (different colors indicate atoms of different layers) is also shown in (a). DFT-based simulations of STM images of the structure presented in (a) and (b) using the empirical model with (c) a blunt STM tip consisting of 12 atoms and (d) an atomically sharp STM tip consisting of 13 atoms. Only in the latter case, atomic resolution is visible on both the cluster edges and on the top facet of the cluster. Dashed lines are added to $(\mathrm{a}-\mathrm{c})$ as guides for the eye to highlight the lateral broadening of the cluster by tip convolution.

Upon comparison of simulated STM images of the cluster on bare $\mathrm{Au}(111)$ and on $\mathrm{NaCl}-\mathrm{Au}(111)$ with the same STM tip [see Fig. 4(c) and (d)], we find that the resolution is similar in both cases, i.e. with and without the $\mathrm{NaCl}$ layer surrounding the cluster. This implies that the observed enhancement of the resolution cannot be related to the modified tunneling conditions between tip and sample upon adding the $\mathrm{NaCl}$ layer. In particular, the $\mathrm{NaCl}$ layer significantly lowers the work function of the $\mathrm{Au}(111)$ surface. ${ }^{\mathbf{1 7}}$ Moreover, the STM tip is located closer to the NaCl-Au(111) surface when compared to the $\mathrm{Au}(111)$ surface under the same tunneling conditions, which results in the lower apparent height of the $\mathrm{NaCl}$ film in STM topography 
images. According to our simulations, however, imaging of the Co cluster surface appears to remain largely unaffected.

Although the model does not allow us to take into account the specific chemical nature of the terminating $\mathrm{Cl}$ atom, the calculations clearly illustrate that an atomically sharp apex is required to resolve the atomic structure of the top facet of the cluster [Fig. 3(c)], as demonstrated in the simulated STM images in Fig. 4(c) and (d). Furthermore, in order to simulate the effect of a tip dominated by the presence of a single $\mathrm{Cl}$ atom picked up from the surface, we also performed calculations with a tip composed of a single atom and found that the resulting simulated image is similar to that obtained employing an extended tip that is composed of 13 atoms and that is terminated by a single atom [see Fig. 4(d)]. The increased resolution on Co clusters on $\mathrm{NaCl}-\mathrm{Au}(111)$ must therefore be related to a different tip apex, which is obtained by picking up a $\mathrm{Cl}^{-}$ion when scanning the locally damaged $\mathrm{NaCl}$ film. ${ }^{15-17}$

\section{Conclusion}

We investigated the morphology and electronic behavior of Co clusters deposited on an insulating $\mathrm{NaCl}$ layer on $\mathrm{Au}(111)$. Due to the (partial) penetration of the clusters in the $\mathrm{NaCl}$ film, the majority of the clusters is in metallic contact with the supporting $\mathrm{Au}(111)$ surface as is evidenced by the disappearance of the Coulomb blockade in the STS spectra. The full atomic structure of the Co cluster surface is probed by high-resolution STM imaging using a functionalized tip that can be conveniently obtained by scanning on the surrounding $\mathrm{NaCl}$ layer. Relying on a DFT-based model for simulating STM images, we demonstrate the role of the size and shape of the STM tip apex in the visualization of Co clusters on NaCl-Au(111). Our findings open new perspectives for atomic structure determination with STM of clusters and molecules deposited on surfaces.

\section{Acknowledgements}

This research has been supported by the Research Foundation - Flanders (FWO, Belgium), the KU Leuven Research Fund (GOA program), and the ERC-AG SEPON project. K. L. acknowledges the Institute for the Promotion of Innovation through Science and Technology in Flanders (IWT-Vlaanderen). K. S. is a post-doctoral researcher of the FWO. Networking from the COST Action MP0903 is gratefully acknowledged.

\section{Notes and references}

1 J. V. Lauritsen, R. T. Vang and F. Besenbacher, Catal. Today, 2006, 111, 34.

2 D. V. Potapenko, J. Hrbek and R. M. Osgood, ACS Nano, 2008, 2, 1353.

3 C. Barth, M. Gingras, A. S. Foster, A. Gulans, G. Félix, T. Hynninen, R. Peresutti and C. R. Henry, Adv. Mater., 2012, 24, 3228.

4 Y. Han, D. S. He, Y. Liu, S. Xie, T. Tsukuda and Z. Y. Li, Small, 2012, 8, 2361.
5 S. Bals, S. Van Aert, C. P. Romero, K. Lauwaet, M. J. Van Bael, B. Schoeters, B. Partoens, E. Yücelen, P. Lievens and G. Van Tendeloo, Nat. Commun., 2012, 1887, 1.

6 P. M. Albrecht and J. W. Lyding, Small, 2007, 3, 146.

7 I. Swart, T. Sonnleitner, J. Niedenführ and J. Repp, Nano Lett., 2012, 12, 1270.

8 A. A. Herzing, C. J. Kiely, A. F. Carley, P. Landon and G. J. Hutchings, Science, 2008, 321, 1331.

9 Z. Y. Li, N. P. Young, M. Di Vece, S. Palomba, R. E. Palmer, A. L. Bleloch, B. C. Curley, R. L. Johnston, J. Jiang and J. Yuan, Nature, 2008, 451, 46.

10 S. Van Aert, K. J. Batenburg, M. D. Rossell, R. Erni and G. Van Tendeloo, Nature, 2011, 470, 374.

11 A. Piednoir, E. Perrot, S. Granjeaud, A. Humbert, C. Chapon and C. R. Henry, Surf. Sci., 1997, 391, 17.

12 E. J. Lautenschlager and R. E. Martinez, Chem. Phys. Lett., 2001, 341, 207.

13 N. Isomura, X. Wu and Y. Watanabe, J. Chem. Phys., 2009, 131, 164707.

14 D. Alloyeau, C. Mottet and C. Ricolleau, Nanoalloys: Synthesis, Structure and Properties, Springer-Verlag, London, 2012.

15 L. Gross, F. Mohn, N. Moll, P. Liljeroth and G. Meyer, Science, 2009, 325, 1110.

16 L. Gross, N. Moll, F. Mohn, A. Curioni, G. Meyer, F. Hanke and M. Persson, Phys. Rev. Lett., 2011, 107, 086101.

17 K. Lauwaet, K. Schouteden, E. Janssens, C. Van Haesendonck, P. Lievens, M. I. Trioni, L. Giordano and G. Pacchioni, Phys. Rev. B: Condens. Matter Mater. Phys, 2012, 85, 245440.

18 The laser vaporization source produces Co clusters with a kinetic energy of around $0.15 \mathrm{eV}$ per atom, which is small when compared to the atomic binding energy within the cluster. Therefore limited cluster fragmentation is expected upon impact, ${ }^{25,38}$ as can be concluded from the overall shape of the height histograms in Fig. 1(c). The discrepancy between the STM based height distributions of deposited clusters and the diameter distribution of gas phase clusters in the height range $<1.0 \mathrm{~nm}$ might indicate fragmentation of a minor fraction of the clusters.

19 This distribution is derived from the recorded mass spectrum assuming a spherical shape and using the cobalt Wigner-Seitz radius of $0.138 \mathrm{~nm}$ as the representative radius of a single atom in the cluster.

20 K. Lauwaet, K. Schouteden, E. Janssens, C. Van Haesendonck and P. Lievens, J. Phys. Condens. Matter, 2012, 85, 245440.

21 The gap probed in $I(V)$ spectra such as spectrum (3) in Fig. 2(a) can be identified as the region of zero tunneling current. The size of the gap $V_{\text {gap }}$ is determined by fitting the quasi linear $I(V)$ behavior at the left and right hand side of the gap using a straight line [lines are not shown in Fig. 2(a)]; the voltages at which these two lines cross with the horizontal axis at zero tunneling current determine the gap size.

22 L. P. Kouwenhoven, C. M. Marcus, P. L. McEuen, S. Tarucha, R. M. Westervelt and N. S. Wingreen, Mesoscopic Electron Transport, 1997, vol. 345, p. 16. 
$23 V_{\text {gap }}$ is mainly determined by the dominating capacitance formed by the two junctions, i.e., $V_{\text {gap }}=e / \max \left\{C_{1}, C_{2}\right\}^{22}$ with e being the electron charge and $C_{1}$ and $C_{2}$ the capacitance of cluster-tip and cluster-substrate, respectively. Using $\varepsilon_{\mathrm{NaCl}}=5.9,{ }^{39} C_{1}$ and $C_{2}$ can be estimated via the parallel plate model: $C=\varepsilon_{0} \varepsilon_{\mathrm{r}} S / d$, with $S$ being the surface area of the two electrodes that form the capacitance and $d$ the distance between them. Considering the small thickness and the large relative permittivity of the NaCl layer, $C_{2}$ is typically larger than $C_{1}$.

24 W. Hebenstreit, J. Redinger, Z. Horozova, M. Schmid, R. Podloucky and P. Varga, Surf. Sci., 1999, 424, L321.

25 Q. Hou, M. Hou, L. Bardotti, B. Prevel, P. Mélinon and A. Pérez, Phys. Rev. B: Condens. Matter Mater. Phys., 2000, 62, 2825.

26 K. Schouteden, A. Lando, E. Janssens, C. Van Haesendonck and P. Lievens, New J. Phys., 2008, 10, 083005.

27 O. Kitakami, H. Sato, Y. Shimada, F. Sato and M. Tanaka, Phys. Rev. B: Condens. Matter Mater. Phys., 1997, 56, 13849.

28 S. Rives, A. Catherinot, F. Dumas-Bouchiat, C. Champeaux, A. Videcoq and R. Ferrando, Phys. Rev. B: Condens. Matter Mater. Phys., 2008, 77, 085407.

29 K. Schouteden, P. Lievens and C. Van Haesendonck, Phys. Rev. B: Condens. Matter Mater. Phys., 2009, 79, 195409.

30 I. Horcas, R. Fernandez, J. M. Gomez-Rodriguez, J. Colchero, J. Gomez-Herrero and A. M. Baro, Rev. Sci. Instrum., 2007, 78, 013705.

31 W. Bouwen, P. Thoen, F. Vanhoutte, S. Bouckaert, F. Despa, H. Weidele, R. E. Silverans and P. Lievens, Rev. Sci. Instrum., 2000, 71, 54 .

32 K. Schouteden, Y.-J. Zeng, K. Lauwaet, C. P. Romero, B. Goris, S. Bals, G. Van Tendeloo, P. Lievens and C. Van Haesendonck, Nanoscale, 2013, 5, 3757.
33 J. P. Perdew, K. Burke and M. Ernzerhof, Phys. Rev. Lett., 1996, 77, 3865.

34 P. Giannozzi, S. Baroni, N. Bonini, M. Calandra, R. Car, C. Cavazzoni, D. Ceresoli, G. Chiarotti, M. Cococcioni, I. Dabo, A. Dal Corso, S. Fabris, G. Fratesi, S. de Gironcoli, R. Gebauer, U. Gerstmann, C. Gougoussis, A. Kokalj, M. Lazzeri, L. MartinSamos, N. Marzari, F. Mauri, R. Mazzarello, S. Paolini, A. Pasquarello, L. Paulatto, C. Sbraccia, S. Scandolo, G. Sclauzero, A. P. Seitsonen, A. Smogunov, P. Umari and R. M. Wentzcovitch, J. Phys.: Condens. Matter, 2009, 21, 39552.

35 J. Tersoff and D. R. Hamann, Phys. Rev. B: Condens. Matter Mater. Phys., 1985, 81, 805.

36 The parameters $A_{j}$ and $B_{j}$ are derived from an analysis of DFT-simulated STM images assuming a proportionality of the Tersoff-Hamann images to the tunneling current. The following values are so derived: $B_{j}^{\mathrm{Au}}=2.03 \AA^{-1}, B_{\mathrm{Co}}^{j}=2.32$ $\AA^{-1}, B_{\mathrm{Cl}}^{j}=3.00 \AA^{-1}, A_{\mathrm{Au}}^{j}=A_{\mathrm{Co}}^{j}=1.0$ and $A_{\mathrm{Cl}}^{j}=0.01$. The value of the isosurface in the DFT calculations was chosen such that the apparent height of the NaCl layer on $\mathrm{Au}(111)$ is in the 0.4 to $0.5 \mathrm{~nm}$ range, in correspondence to the experimental observations. ${ }^{21}$

37 W. Xu, J. G. Wang, M. F. Jacobsen, M. Mura, M. Yu, R. E. A. Kelly, Q. Q. Meng, E. Laegsgaard, I. Stensgaard, T. R. Linderoth, J. Kjems, L. N. Kantorovich, K. V. Gothelf and F. Besenbacher, Angew. Chem. Int. Ed, 2010, 49, 9373.

38 H. Haberland, Z. Insepov and M. Moseler, Phys. Rev. B, 1995, 51, 11061.

39 C. Andeen, J. Fontanella and D. Schuele, Phys. Rev. B, 1970, 2, 5068.

40 K. Schouteden, A. Volodin, Z. Li and C. Van Haesendonck, Carbon, 2013, 61, 379.

41 B. Voigtländer, G. Meyer and N. M. Amer, Phys. Rev. B, 1991, 44, 10354. 